COMMUNICATIONS IN

ANALYSIS AND GEOMETRY

Volume 6, Number 1, 153-172, 1998

\title{
Calabi-Yau threefolds with positive second Chern class
}

\author{
Keiji Oguiso and Thomas Peternell
}

\begin{abstract}
The second Chern class of a Calabi-Yau threefold is called positive if, regarded as a linear form on the Picard group, it takes positive values on the nef cone, that is, the closure of the ample cone, except the origin. The purpose of this paper is to show that every CalabiYau threefold with positive second Chern class admits only finitely many different proper algebraic fiber space structures. We also give some partial results on the finiteness of birational contractions. It is also shown that any smooth Calabi-Yau threefold in a smooth Fano fourfold has positive second Chern class.
\end{abstract}

\section{Introduction.}

In connection with the mirror phenomenon expected for Calabi-Yau threefolds, D.Morrison ([14]) raised the following

Cone Conjecture. In the nef cone $\overline{A m p}(X)$ of a Calabi-Yau threefold $X$, there exists a finite rational polyhedral cone $\Pi$ such that the union of the translates $\gamma(\Pi)$ by automorphisms $\gamma \in \operatorname{Aut}(X)$ covers the convex hull of the rational points lying in the nef cone $\overline{A m p}(X)$.

However, at the present time, this conjecture has been checked only for one non-trivial example by [7] and very little are known in general.

On the other hand, in the course of his classification program of CalabiYau threefolds according to the behaviour of the second Chern class, Wilson ([26]) observed that the automorphism group of a Calabi-Yau threefold $X$, which is in general a discrete group, is actually finite if the second Chern class is positive, that is, $c_{2}(X) \cdot D>0$ for all non-zero nef divisors $D$, and then asked :

Question 1. Is the nef cone of a Calabi-Yau threefold with positive second Chern class a finite rational polyhedral cone? 
An important theorem of Miyaoka [13] shows that the second Chern class is non-negative for every Calabi-Yau threefold $X$, that is, $c_{2}(X) \cdot D \geq 0$ for all nef divisors $D$ on $X$. Since it is also known that every (non-zero) rational point of the nef cone of a Calabi-Yau threefold with positive second Chern class gives rise to an (algebraic) fiber space structure for $X([24,17,26])$, this question is translated into the following more geometrical (but slightly weaker)

Question 2. Are there only finitely many different algebraic fiber space structures on a given Calabi-Yau threefold with positive second Chern class?

The main purpose of this paper is to study Question 2. Here and throughout this paper, a Calabi-Yau threefold means a normal projective complex threefold $X$ with at most $\mathbb{Q}$-factorial terminal singularities, with $\omega_{X}:=\mathcal{O}_{X}\left(K_{X}\right) \simeq \mathcal{O}_{X}$ and with $H^{1}\left(X, \mathcal{O}_{X}\right)=0$. An (algebraic) fiber space structure on $X$ is a morphism $\Phi: X \rightarrow Y$ onto a normal projective variety $Y$ with connected fibers. We call such $\Phi: X \rightarrow Y$ a proper algebraic fiber space if $0<\operatorname{dim} Y<\operatorname{dim} X$ and a birational contraction if $\operatorname{dim} Y=\operatorname{dim} X$.

Our Main Theorem is as follows. (See also the remark at the end of section 3 for a slight generalization.)

Main Theorem 1. Every Calabi-Yau threefold with positive second Chern class admits only finitely many proper algebraic fiber space structures, that is, such a Calabi-Yau threefold has only finitely many different K3 fibrations and elliptic fibrations.

It is known that there is a Calabi-Yau threefold (with non-positive second Chern class) which has infinitely many different K3 fibrations and elliptic fibrations ([17]).

We also give some partial results concerning the finiteness of birational contractions (Propositions 4.1 and 4.6). These together with Wilson's observation quoted before provide another evidence for the Cone Conjecture.

Our proof of main Theorem $1(\S 3)$ is based on the following topics :

(1) Alexeev's theory on log surfaces $([1,23])$

(2) Estimates of global log canonical indices of the base spaces of certain elliptic fiber spaces (Proposition 2.4) after Kodaira, Ueno, Kawamata and Nakayama.

In the last section of this paper we also study second Chern classes of smooth Calabi-Yau threefolds in smooth Fano fourfolds, that is, smooth anti-canonical members of Fano fourfolds. 
Main Theorem 2. Every smooth Calabi-Yau threefold in a smooth Fano fourfold has positive second Chern class. In particular, any smooth CalabiYau threefolds in a Fano fourfold do not admit abelian fibrations.

Our proof is an application of the following remarkable Theorem due to Kollár and several structure theorems of fibered Calabi-Yau threefolds ([17, $21,18])$.

Theorem ([4, appendix $])$. Let $V$ be a Fano manifold with $n=\operatorname{dim} V \geq$ 4. Let $X \in\left|-K_{V}\right|$ be a smooth member. Then the inclusion map

$$
i_{*}: \overline{N E}(X) \rightarrow \overline{N E}(V)
$$

is bijective. In particular, the ample cone of a smooth Calabi-Yau threefold in a Fano fourfold is a finite rational polyhedral cone.

Combining these with Wilson's observation [26], we also obtain

Corollary. The automorphism group of a smooth Calabi-Yau threefold in a Fano fourfold is finite.

Each of these three properties gives an obstruction for a smooth CalabiYau threefold to be embedded in a smooth Fano fourfold. It is also interesting to note that this is not the case for $\mathrm{K} 3$ surfaces. For example, it is known by [22] that the Fermat quartic surface in $\mathbb{P}^{3}$ has an infinite automorphism group.

Acknowledgements. The first author would like to express his thanks to the Deutsche Forschungsgemeinschaft for financial support during his stay at the University of Bayreuth in September 1994 when the main part of this joint work was done and also to Professor J. Kollár for financial support during his stay in Utah, July 1994, where he learned the very interesting theory of Alexeev. This plays an essential role in this paper. Both authors would like to express their thanks to Doctor D. Dais for useful discussions and Professor T. Katsura for pointing out a serious gap in the proof of main Theorem 2 in a preliminary version of this paper.

\section{Preliminaries.}

We shall employ freely the standard notation of the theory of minimal model program as given in [11]. We start stating several easy remarks in order to make our arguments clear. 
Definition (1.1). (1) A normal projective complex threefold $X$ with at most $\mathbb{Q}$-factorial terminal singularities is called a Calabi-Yau threefold if $\omega_{X}:=\mathcal{O}_{X}\left(K_{X}\right) \simeq \mathcal{O}_{X}$ and $H^{1}\left(X, \mathcal{O}_{X}\right)=0$. Note that $X$ has then at most isolated rational singularities ([20]).

(2) The second Chern class $c_{2}(X)$ of a Calabi-Yau threefold $X$ is defined by $c_{2}(X):=\nu_{*} c_{2}(Y)$, where $\nu: Y \rightarrow X$ is a resolution of $\operatorname{Sing}(X)$. This well-defined cycle is regarded as a linear form on the Picard group. The second Chern class $c_{2}(X)$ of a Calabi-Yau threefold $X$ is called positive, or $c_{2}(X)>0$ for short, if $c_{2}(X)$ takes positive values on the nef cone $\overline{A m p}(X)-\{0\}$ of $X$.

(3) By an algebraic fiber space, or an algebraic contraction, we mean a surjective morphism $\Phi: X \rightarrow Y$ onto a normal projective variety $Y$ with connected fibers. An algebraic fiber space $\Phi: X \rightarrow Y$ is called proper if $0<\operatorname{dim}(Y)<\operatorname{dim}(X)$ and a birational contraction if $\operatorname{dim}(Y)=\operatorname{dim}(X)$. A birational contraction $\varphi: X \rightarrow Y$ is called primitive if it cannot be factored into several of those contractions.

(4) By a Fano $n$-fold, we mean a smooth projective complex $n$-fold $V$ whose anti-canonical divisor $-K_{V}$ is ample. It is well known that every Fano $n$-fold is simply connected. In particular, if $X$ is a smooth member of $\left|-K_{V}\right|$ of a Fano fourfold $V$, then $X$ is a (smooth simply connected) Calabi-Yau threefold.

Now we apply several semi-ampleness criteria $([24,17,26])$ to obtain

Lemma (1.2). Let $X$ be a Calabi-Yau threefold with $c_{2}(X)>0$. Then every $\mathbb{Q}$-divisor $D$ lying in the intersection of the nef cone and the cubic cone $\left(D^{3}=0\right)$ gives rise to a proper algebraic fiber space $\Phi_{|m D|}: X \rightarrow W$ for some positive integer $m$. The same holds if $D$ is nef and big $\left(D^{3}>0\right)$, only that now the associated morphism is a birational contraction.

Lemma (1.3). Let $C \subset \mathbb{R}^{\rho}=\mathbb{Z}^{\rho} \otimes_{\mathbb{Z}} \mathbb{R}$ be a closed cone with vertex 0 . Suppose that there is a linear functional $L: \mathbb{R}^{\rho} \rightarrow \mathbb{R}$ such that $L$ is positive on $C \backslash\{0\}$. Let $N$ be a positive real number and let $C_{N}=C \cap\left\{x \in \mathbb{R}^{\rho} \mid L(x) \leq\right.$ $N\}$. Then $C_{N}$ contains only finitely many lattice points.

Proof. This follows from the compactness of $C_{N}$.

We shall apply this lemma in the following two special cases. 
Corollary (1.4). Let $X$ be a Calabi-Yau threefold with $c_{2}(X)>0$. Then

$$
\overline{A m p}(X) \cap\left\{x \in P i c(X) \mid c_{2}(X) \cdot x \leq N\right\}
$$

is finite for any given positive number $N$.

Corollary (1.5). Let $(X, H)$ be a polarised normal variety. Then the number of numerical equivalence classes of effective curves $l$ satisfying $l \cdot H \leq N$ is finite for every positive number $N$.

The next lemma and its corollary will frequently be used in section 2 .

Lemma (1.6). Let $\mu: T \rightarrow S$ be a birational morphism between normal varieties $T$ and $S$ and $D$ an (integral) Weil divisor on $T$. Then there is a natural isomorphism

$$
\left(\mu_{*}\left(\mathcal{O}_{T}(D)\right)\right)^{* *} \simeq \mathcal{O}_{S}\left(\mu_{*}(D)\right),
$$

where $\mu_{*}$ on the right hand side denotes pushforward of cycles. In particular, if $\mu_{*}\left(\mathcal{O}_{T}(D)\right)$ is invertible, then $\mu_{*}\left(\mathcal{O}_{T}(D)\right) \simeq \mathcal{O}_{S}\left(\mu_{*}(D)\right)$.

Proof. Since the sheaves $\left(\mu_{*}\left(\mathcal{O}_{T}(D)\right)\right)^{* *}$ and $\mathcal{O}_{S}\left(\mu_{*}(D)\right)$ coincide outside a set of codimension at least 2 and since both are reflexive, they must coincide on $S$.

Corollary (1.7). Let $\mu: T \rightarrow S$ be as in (1.6). Let $\mathcal{L}$ be a reflexive sheaf of rank 1 on $T$. Then the following two statements are equivalent;

(1) $\mu_{*}(\mathcal{L}) \simeq \mathcal{O}_{S}$

(2) there is an effective integral Weil divisor $E$ (possibly 0 ) supported in the exceptional locus of $\mu$ such that $\mathcal{L} \simeq \mathcal{O}_{T}(E)$.

2. Log canonical index of the base spaces of certain elliptic fibrations.

Until the Remark of Proposition (2.4), we work in the following setting:

Setting (2.1). Let $\varphi: Y \rightarrow S$ be a proper surjective morphism between normal projective varieties $Y$ and $S$ such that

(1) $\operatorname{dim} Y=\operatorname{dim} S+1=n+1$; 
(2) $Y$ has only terminal singularities of index 1 ;

(3) $\omega_{Y} \simeq \varphi^{*}(\mathcal{L})$ with an invertible sheaf $\mathcal{L}$ which is globally generated in codimension 1 on $S$, that is, the codimension of $\operatorname{Supp}\left(\operatorname{Coker}\left(H^{0}(\mathcal{L}) \otimes\right.\right.$ $\left.\mathcal{O}_{S} \rightarrow \mathcal{L}\right)$ ) is at least 2 ;

(4) the geometric generic fiber $Y_{\bar{\eta}}$ is a smooth elliptic curve.

By a big open set we mean a Zariski open set whose complement has codimension at least 2 . We first point out the following

Lemma (2.2). There is a big open set $S^{0} \subset S$ such that every schemetheoretic fiber $Y_{s}$ over $s \in S^{0}$ is one-dimensional and not multiple as a 1 -cycle.

Proof. Choose $n-1$ general very ample divisors $H_{1}, \ldots, H_{n-1}$ on $S$ such that their intersection $C=H_{1} \cap \ldots \cap H_{n-1}$ is a smooth irreducible curve. Then consider the induced elliptic surface $\varphi \mid F: F=Y \times{ }_{S} C \rightarrow C$. Note that $F$ is smooth if $H_{1}, \ldots, H_{n-1}$ are general and that the linear system $\left|K_{F}\right|$ is free by the condition (3) in (2.1) and the adjunction formula. In particular, $\varphi \mid F$ has no multiple fibers. This implies the result.

(2.3). Following Nakayama ([16]), let us consider the next commutative diagram:

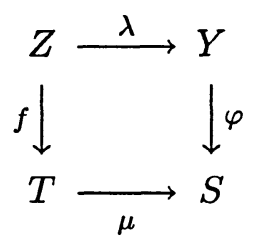

where,

(1) $Z$ and $T$ are nonsingular,

(2) $\lambda$ and $\mu$ are surjective birational morphisms,

(3) $f: Z \rightarrow T$ is a (projective) elliptic fibration, which is smooth over a Zariski open subset $T^{0}$ of $T$ whose complement $D:=T-T^{0}$ is a simple normal crossing divisor, and

(4) $\mu \mid T^{0}: T^{0} \rightarrow \mu\left(T^{0}\right)$ is isomorphic.

Then, we deduce by [9] that 
(5) the usual $J$-function $J: T^{0} \longrightarrow \mathbb{C}$ extends to a morphism $J: T \longrightarrow$ $\mathbb{P}^{1}$, and

(6) $f_{*} \omega_{Z}$ is an invertible sheaf with $\left(f_{*} \omega_{Z}\right)^{\otimes 12} \simeq \omega_{T}^{\otimes 12} \otimes \mathcal{O}_{T}\left(\Sigma 12 a_{j} D_{j}+H\right)$,

where $D_{j}$ are divisors supported in $D$, the $a_{j}$ are rational numbers with $12 a_{j} \in\{0,2,3,4,6,8,9,10\}$ according to the type of general (singular) fibers over $D_{j}$, and $H$ is a general element in $\left|J^{*} \mathcal{O}_{\mathbb{P}^{1}}(1)\right|$.

Here, we do not need the condition (3) in (2.1). From now, we use the condition (3) in (2.1).

Note that each irreducible component of $D$ over which a general fiber of $f$ is multiple is contracted by $\mu$ to some algebraic set whose codimension in $S$ is at least two by (2.2). Now, we can apply [16] to obtain

(7) the pair $\left(S, \Delta_{S}\right)$ is a log variety with only $\log$ terminal singularities, where the divisor $\Delta_{S}$ is defined by

(8) $\Delta_{S}=\mu_{*}\left(\frac{1}{12} H+\sum a_{j} D_{j}\right)$.

The main point of this section is

Proposition (2.4). In the notation of (2.1)-(2.3) we have $\mathcal{O}_{S}\left(12\left(K_{S}+\right.\right.$ $\left.\left.\Delta_{S}\right)\right) \simeq \mathcal{L}^{\otimes 12}$. In particular, the integral Weil divisor $12\left(K_{S}+\Delta_{S}\right)$ is Cartier.

Proof. First we show

Claim (2.5). There is an effective Cartier divisor $E$ supported on the exceptional locus of $\mu$ such that $f_{*}\left(\omega_{Z}\right) \simeq \mathcal{O}_{T}(E) \otimes \mu^{*}(\mathcal{L})$.

Proof of (2.5). By (2.1)(2) we have $\omega_{Y}=\lambda_{*}\left(\omega_{Z}\right)$. Therefore the projection formula yields

$$
\mu_{*}\left(f_{*}\left(\omega_{Z}\right)\right)=\varphi_{*}\left(\lambda_{*}\left(\omega_{Z}\right)\right)=\varphi_{*}\left(\omega_{Y}\right)=\varphi_{*} \varphi^{*}(\mathcal{L})=\mathcal{L} .
$$

Consequently $\mu_{*}\left(f_{*}\left(\omega_{Z}\right) \otimes \mu^{*}\left(\mathcal{L}^{-1}\right)\right) \simeq \mathcal{O}_{S}$. Now the claim (2.5) follows from (1.7).

Coming back to the proof of (2.4), we calculate by virtue of (2.5) and $(2.3)(6)$ :

$$
\mathcal{O}_{T}(12 E) \otimes \mu^{*}\left(\mathcal{L}^{\otimes 12}\right) \simeq\left(f_{*}\left(\omega_{Z}\right)\right)^{\otimes 12}
$$




$$
\simeq \omega_{T}^{\otimes 12} \otimes \mathcal{O}_{T}(H) \otimes \mathcal{O}_{T}\left(12 \sum a_{j} D_{j}\right)=\mathcal{O}_{T}\left(12 K_{T}+H+12 \sum a_{j} D_{j}\right) .
$$

Using (1.6), (1.7) and the equality of Weil divisors $\mu_{*}\left(K_{T}\right)=K_{S}$, we conclude

$$
\begin{aligned}
\mathcal{L}^{\otimes 12} & =\mu_{*}\left(\mathcal{O}_{T}(12 E) \otimes \mu^{*}\left(\mathcal{L}^{\otimes 12}\right)\right) \simeq \mu_{*}\left(\mathcal{O}_{T}\left(12 K_{T}+H+12 \sum a_{j} D_{j}\right)\right) \\
& \simeq \mathcal{O}_{S}\left(\mu_{*}\left(12 K_{T}+H+12 \sum a_{j} D_{j}\right)\right)=\mathcal{O}_{S}\left(12\left(K_{S}+\Delta_{S}\right)\right) .
\end{aligned}
$$

This completes the proof of (2.4).

Remark. This proof also shows that $\left(S, \Delta_{S}\right)$ has only log terminal singularities (cf. [16]).

We now return to our study of Calabi-Yau threefolds. This might be the right place to introduce the following notion due to V. Alexeev $[1,23]$.

Definition. Let $(S, \Delta)$ be a log surface with only log terminal singularities. Write $\Delta=\sum a_{j} \Delta_{j}$. Let $\nu: S^{\prime} \rightarrow S$ be the minimal resolution with exceptional divisor $\sum E_{j}$. Let $\Delta_{j}^{\prime}$ denote the strict transform of $\Delta_{j}$ in $S^{\prime}$ and set

$$
K_{S^{\prime}}+\sum a_{j} \Delta_{j}^{\prime}+\sum E_{i}=\nu^{*}\left(K_{S}+\sum a_{j} \Delta_{j}\right)+\sum \alpha_{i} E_{i} .
$$

Here $\alpha_{i}$ are (uniquely determined) rational numbers. Then $(S, \Delta)$ is said to be $\epsilon-$ MRS $\log$ terminal if $\alpha_{i}>\epsilon$ and if $0 \leq a_{j}<1-\epsilon$ for all $i, j$.

Corollary (2.6). Let $X$ be a Calabi-Yau threefold. Suppose that $X$ has an elliptic fiber space structure $\varphi: X \rightarrow W$. Then $\mathcal{O}_{W}\left(12\left(K_{W}+\Delta_{W}\right)\right) \simeq \mathcal{O}_{W}$, where $\Delta_{W}$ is defined as in (2.3)(8). In particular, the log surface $\left(W, \Delta_{W}\right)$ is $\frac{1}{13}-M R S \log$ terminal.

Proof. Since $\omega_{X} \simeq \mathcal{O}_{X} \simeq \varphi^{*}\left(\mathcal{O}_{W}\right)$, the first part follows from (2.4). Note that $\alpha_{i}>0$ because $\left(W, \Delta_{W}\right)$ is log terminal. Now the second statement follows from an immediate calculation.

We shall close this section by showing the next Lemma used in the proof of main Theorem 2.

Lemma (2.7). Let $\phi: S \rightarrow C$ be a relatively minimal elliptic surface. Assume that $\phi$ is a smooth morphism, that is, $\phi$ has no singular fibers. 
Then, there is an étale cover $\pi: \tilde{C} \rightarrow C$ such that the induced fibration $\tilde{\phi}: \tilde{S}:=S \times_{C} \tilde{C} \rightarrow \tilde{C}$ satisfies $h^{1}\left(\mathcal{O}_{\tilde{S}}\right)=h^{1}\left(\mathcal{O}_{\tilde{C}}\right)+1$. Moreover, we can choose such $\pi$ as an (étale) cyclic Galois covering of order I with I|12 (possibly $I=1$ ).

Proof. Set $\mathcal{L}=\phi_{*}\left(\omega_{S / C}\right)$ as in (2.3). Then, $\mathcal{L}$ is an invertible sheaf on $C$ with $\omega_{S} \simeq \phi^{*}\left(\omega_{C} \otimes \mathcal{L}\right)$ by the canonical bundle formula for an elliptic surface ([3]). Moreover, the smoothness of $\phi$ implies $\mathcal{L}^{\otimes 12} \simeq \mathcal{O}_{C}$. In fact, the formula in (2.3)(6) shows $h^{0}\left(\mathcal{L}^{\otimes 12}\right) \neq 0$, while we have $\operatorname{deg} \mathcal{L}=0$ by the smoothness of $\phi$. Let $n$ be the smallest positive integer such that $\mathcal{L}^{\otimes n} \simeq \mathcal{O}_{C}$ and consider the associated étale Galois covering

$$
\pi: \tilde{C}:=\operatorname{Spec}_{\mathcal{O}_{C}}\left(\oplus_{i=0}^{n-1} \mathcal{L}^{-i}\right) \rightarrow C .
$$

Then, $\tilde{C}$ is a smooth irreducible curve with $\pi^{*} \mathcal{L} \simeq \mathcal{O}_{\tilde{C}}$. Set $\tilde{S}:=S \times_{C} \tilde{C}$. Let $\tilde{\pi}: \tilde{S} \rightarrow S$ and $\tilde{\phi}: \tilde{S} \rightarrow \tilde{C}$ be the natural morphisms. Since $\pi$ and $\tilde{\pi}$ are étale, we calculate,

$$
\omega_{\tilde{S}}=\tilde{\pi}^{*}\left(\omega_{S}\right)=\tilde{\pi}^{*} \phi^{*}\left(\omega_{C} \otimes \mathcal{L}\right)=\tilde{\phi}^{*} \pi^{*}\left(\omega_{C} \otimes \mathcal{L}\right)=\tilde{\phi}^{*}\left(\omega_{\tilde{C}}\right)
$$

Using this equality and the Serre duality, we get

$$
h^{2}\left(\mathcal{O}_{\tilde{S}}\right)=h^{0}\left(\omega_{\tilde{S}}\right)=h^{0}\left(\tilde{\phi}^{*} \omega_{\tilde{C}}\right)=h^{0}\left(\tilde{\phi}_{*} \tilde{\phi}^{*}\left(\omega_{\tilde{C}}\right)\right)=h^{0}\left(\omega_{\tilde{C}}\right)=h^{1}\left(\mathcal{O}_{\tilde{C}}\right) .
$$

Combining this with the Noether's formula, we get

$$
1-h^{1}\left(\mathcal{O}_{\tilde{S}}\right)+h^{1}\left(\mathcal{O}_{\tilde{C}}\right)=\chi\left(\mathcal{O}_{\tilde{S}}\right)=(1 / 12)\left(K_{\tilde{S}}^{2}+c_{2}(\tilde{S})\right)=0 .
$$

Here, the last equality follows from the fact that $\tilde{\phi}: \tilde{S} \rightarrow \tilde{C}$ is also a smooth elliptic fibration. Thus, $h^{1}\left(\mathcal{O}_{\tilde{S}}\right)=h^{1}\left(\mathcal{O}_{\tilde{C}}\right)+1$.

Remark. There is a relatively minimal elliptic surface $\phi: S \rightarrow C$ with no singular fibers but with $h^{1}\left(\mathcal{O}_{S}\right)=h^{1}\left(\mathcal{O}_{C}\right)$. In fact, the Albanese map of a hyperelliptic surface gives such an elliptic fibration.

\section{Proof of Main Theorem 1.}

In this section we shall prove Main Theorem 1. Throughout this section, $X$ is assumed to be a Calabi-Yau threefold with $c_{2}(X)>0$. According to $[24,17,26]$, every possible proper fiber space structure $\varphi: X \rightarrow W$ is one of the following: 
Case 1. $W=\mathbb{P}^{1}$ and the general fiber is a $\mathrm{K} 3$ surface;

Case 2. $\varphi$ is an elliptic fibration with $\Delta_{W}>0$.

We will treat these two cases separately.

Case 1. Choose a general element $F \in\left|\varphi^{*}\left(\mathcal{O}_{\mathbb{P}^{1}}(1)\right)\right|$, that is, a general fiber of $\varphi$. Since $F$ is a K3 surface, we have $24=c_{2}(F)=F \cdot c_{2}(X)$, hence the possible classes of $F$ are finite by (1.4). But $\varphi$ is uniquely determined by the class of $F$, since $\varphi=\Phi_{|F|}$.

Case 2. By (2.6), $\left(W, \Delta_{W}\right)$ is a $\frac{1}{13}-$ MRS log terminal surface with $-\left(K_{W}+\Delta_{W}\right) \equiv 0$ and with $K_{W} \not \equiv 0$. By the theory of Alexeev $[2,23]$, those surfaces fit into some bounded projective family, say $\rho: \mathcal{W} \rightarrow \mathcal{P}$. That is, for every elliptic fiber space structure $\varphi: X \rightarrow W$, there exists a point $p_{W} \in \mathcal{P}$ such that $W \simeq \mathcal{W}_{p_{W}}=\rho^{-1}\left(p_{W}\right)$. Let $\mathcal{H}$ be a $\rho$-very ample line bundle on $\mathcal{W}$. Let $\mathcal{W}_{p}=\rho^{-1}(p)$ and $\mathcal{H}_{p}=\left.\mathcal{H}\right|_{\mathcal{W}_{p}}$. Then the function $h(p)=h^{0}\left(\mathcal{O}_{\mathcal{W}_{p}}\left(\mathcal{H}_{p}\right)\right)$ is bounded, that is, there is a constant $B$ such that $h(p) \leq B$ for all $p \in \mathcal{P}$. Now let $H=\mathcal{H}_{p_{W}}$ and choose a general element $C \in|H|$ in such a way that the restriction of $\varphi$ to $M=\varphi^{-1}(C)$ gives a relatively minimal elliptic surface $\varphi: M \rightarrow C$. Note that $K_{M}=\left.M\right|_{M}$ and $K_{M}^{2}=M^{3}=0$. Thus, by the Noether's formula, we calculate:

$$
c_{2}(X) \cdot M=c_{2}(M)=12 \chi\left(\mathcal{O}_{M}\right) \leq 12\left(1+h^{2}\left(\mathcal{O}_{M}\right)\right) .
$$

Here, the first equality follows from the exact sequence,

$$
0 \rightarrow T_{M} \rightarrow T_{X} \mid M \rightarrow N_{X \mid M} \rightarrow 0 .
$$

On the other hand, the cohomology sequence associated with

$$
0 \rightarrow \mathcal{O}_{X}(-M) \rightarrow \mathcal{O}_{X} \rightarrow \mathcal{O}_{M} \rightarrow 0
$$

gives

$$
h^{2}\left(\mathcal{O}_{M}\right) \leq h^{2}\left(\mathcal{O}_{X}\right)+h^{3}\left(\mathcal{O}_{X}(-M)\right)=h^{0}\left(\mathcal{O}_{X}(M)\right)=h^{0}\left(\mathcal{O}_{W}(H)\right) .
$$

Combining these two inequalities, we get,

$$
c_{2}(X) \cdot \varphi^{*}(H)=c_{2}(X) \cdot M \leq 12+12 B .
$$

Hence by (1.4) we obtain the finiteness of the class of $\varphi^{*}(H)$ in the nef cone. Now the finiteness of $\varphi$ follows as in Case 1 .

This completes the proof of Main Theorem 1. 
Remark. Our proof of main Theorem 1 gives also the following statements.

(1) All the rational base spaces of elliptically fibered Calabi-Yau threefolds fit into some bounded projective family. (Compare with [6].)

(2) Let $X$ be any Calabi-Yau threefold. Let $H$ be an ample line bundle on $X$ and $e$ a positive real number. Then there are only finitely many proper algebraic fiber structures on $X$ of the form $\Phi_{|D|}: X \rightarrow W$ with $D \cdot c_{2}(X)>e D \cdot H^{2}$ (cf. [24]).

\section{Divisorial contractions on Calabi-Yau threefolds.}

In this section we are concerned with a primitive divisorial contraction on a Calabi-Yau threefold $X$, that is, a birational contraction $\varphi: X \rightarrow$ $\bar{X}$ contracting exactly a prime divisor $E \subset X$. Then $\bar{X}$ is a Calabi-Yau threefold with canonical singularities along $C=\varphi(E)$. In the literature [25, $26,27,28]$, those contractions are often called of type III if $C$ is a curve and of type II if $C$ is a point, while the small contractions are called of type I. We will keep this notation in this section.

Finiteness in case when $\operatorname{dim} \varphi(E)=0$ is easy :

Proposition (4.1). For any Calabi-Yau threefold $X$, there are only finitely many birational divisorial contractions $\varphi: X \rightarrow \bar{X}$ with dim $\varphi(E)=0$.

Proof. It is sufficient to prove the finiteness for primitive contractions of type II.

Let $\varphi_{i}: X \rightarrow \bar{X}_{i}(i=1,2)$ be two different primitive divisorial birational contractions with exceptional divisors $E_{i}$. Then $E_{i} \cap E_{j}=\emptyset$. In fact, otherwise, consider the curve $B=E_{i} \cap E_{j}$. Let $R_{i} \subset \overline{N E}(X)$ be the extremal rays corresponding to $\varphi_{i}$. Then $[B] \in R_{i}$ for $i=1,2$ so that $R_{i}=R_{j}$, contradiction.

Now let $\varphi_{i}(i \in I)$ be all the primitive contractions of type II in question. Take a general hyperplane section $H \subset X$ and put $F_{i}=E_{i} \cdot H$. Then $F_{i}^{2}<0$ in $H$. Since $F_{i} \cdot F_{j}=0$ for $i \neq j$ by the previous argument, all the $F_{i}$ are linearly independent. Since $\rho(H)<\infty$, we conclude that $I$ must be finite.

From now on we assume that $X$ is a smooth Calabi-Yau threefold with positive second Chern class and consider primitive birational contractions 
of Type $I I I$. Of course, this case is much more difficult than the previous one.

Let $\varphi_{i}: X \rightarrow Y_{i}(i \in I)$ be all the primitive contraction of type III and $E_{i}$ the (prime) exceptional divisor of $\varphi_{i}$. Set $C_{i}:=\varphi_{i}\left(E_{i}\right)$. Then, the general known structure of $p_{i}=\left.\varphi_{i}\right|_{E_{i}}: E_{i} \rightarrow C_{i}$ is as follows ([25, 28]). First, $p_{i}$ is a conic bundle and the base curve $C_{i}$ is always smooth. Moreover, $Y_{i}$ has generically $A_{1}$ - or $A_{2}$ - singularities along $C_{i}$ according whether the general fiber of $p_{i}$ is irreducible or reducible. In the first case $E_{i}$ is a normal surface with at most $A_{n}$ - singularities.

Let $H$ be an ample divisor on $X$. If a divisor $D$ on $X$ is not nef, then the unique positive $t \in \mathbb{R}$ such that $D+t H$ is nef but not ample is called the nef value of $D$ with respect to $H$. In this terminology we have

Lemma (4.2). Let $J$ be a subset of $I$, where $I$ is the set of all primitive contractions of Type III on $X$. Then, $J$ is finite if there are constants $N, M$ such that

(1) $c_{2}(X) \cdot E_{i} \leq N$ for all $i \in J$, and

(2) there is an ample divisor $H$ on $X$ such that the nef values $t_{i}$ of $E_{i}$ with respect to $H$ satisfy $t_{i} \leq M$ for all $i \in J$.

Proof. In fact, consider the set $A=\left\{E_{i}+\left\{t_{i}\right\} H \mid i \in J\right\}$, where $\left\{t_{i}\right\}$ is the round-up of $t_{i}$. Since $\left\{t_{i}\right\} \leq M+1$, it follows from (1) and $c_{2}(X)>0$ that $A$ is contained in a compact set of $\operatorname{Pic}(X) \otimes \mathbb{R}$. Using again (1) we find that the classes of the $E_{i}$ themselves are in a compact set (of $\operatorname{Pic}(X) \otimes \mathbb{R}$ ). Since $h^{0}\left(\mathcal{O}_{X}\left(E_{i}\right)\right)=1$, this gives the finiteness we are looking for.

Lemma (4.3). In the notation of 4.2(2), there is a constant $M$ such that $t_{i} \leq M$ for all those $\varphi_{i}$ for which $-K_{E_{i}}$ is not ample.

Proof. Set $E=E_{i}$ and $\varphi:=\varphi_{i}$. Denote by $j: E \rightarrow X$ the inclusion map. Let $V=j_{*}(\overline{N E}(E)) \subset \overline{N E}(X)$. Since $\varphi$ is primitive, $V$ is a 2-dimensional cone. Let $F$ be a fiber of $\varphi$ and $l$ a cycle on the other geometrically extremal ray of $V$. We fix an ample divisor $H$ on $X$. Clearly the nef value $t$ of $E$ with respect to $H$ is just the nef value of $E \mid E=N_{E}=K_{E}$ with respect to $H \mid E$. Since $N_{E}=K_{E}$ is not negative by our assumption and since $E \cdot F=K_{E} \cdot F=-2$, we must have

$$
E \cdot l \geq 0
$$


by the Kleiman's criterion. On the other hand, the nef value $t$ is computed by

$$
t=\frac{-E \cdot F}{H \cdot F}
$$

or by

$$
t=-\frac{E \cdot l}{H \cdot l}
$$

In the first case we have $t \leq 2$, in the second $t \leq 0$ by $\left({ }^{*}\right)$. In any case, we can take $M=2$.

Proposition (4.4). In the notation of (4.2)(2) there exists a constant $L$ such that $t_{i} \leq L$ for all $\varphi_{i}$ for which $E_{i}$ is normal.

Proof. By (4.3) we may assume that $-K_{E_{i}}$ is ample. Again we shall write $E=E_{i}$ and $\varphi_{i}=\varphi$. Note that either using [25, 28] as cited before or using the classification [5] and [8] and the fact that $E$ is a conic bundle, we see that $E$ is a rational surface with only rational double points as its singularities. In any case, $(E, 0)$ is log terminal. So, by the cone theorem (see [11]) we have

$$
\overline{N E}(E)=\sum \mathbb{R}_{\geq 0}\left[l_{j}\right]
$$

with extremal rational curves $l_{j}$ satisfying $K_{E} \cdot l_{j}=-1$ or -2 . Now we consider as in (4.3) the map $j_{*}: N E(E)=\overline{N E}(E) \rightarrow \overline{N E}(X)$. Take a cycle $l$ on the geometrically extremal ray $\mathrm{R}$ of $V=j_{*}(N E(E))$ not coming from $\varphi$. Write $l \equiv \sum a_{j} l_{j}$ in $N E(E)$ with $a_{j} \geq 0$. Hence the same holds in $V$. But since $l$ is extremal in $V$, we conclude that all those $l_{j}$ for which $a_{j}>0$ (and there are some ) must be in $R$. Hence we may take $l=l_{j}$. In order to prove our claim, we must show that

$$
\frac{-E \cdot l_{j}}{H \cdot l_{j}}
$$

is bounded. But since $-E \cdot l_{j}=-K_{E} \cdot l_{j} \leq 2$, we can take $L=2$.

Remark (4.5). Since $C_{i}$ is smooth, there exists a constant $K$ (depending only on $X$ ) such that $g\left(C_{i}\right) \leq K$ for all $i \in I$. This is proved in [25].

Proposition (4.6). Let $X$ be a smooth Calabi-Yau threefold with positive second Chern class. Then, there are only finitely many primitive birational contractions of Type III such that the divisor $E_{i}$ is smooth and relatively minimal. 
Proof. Let again $E=E_{i}$ and $g=g\left(C_{i}\right)$. Using the exact sequence

$$
0 \rightarrow N_{E \mid X}^{*} \rightarrow \Omega_{X}^{1} \mid E \rightarrow \Omega_{E}^{1} \rightarrow 0,
$$

the relative minimality of $E$ and (4.5), we obtain,

$$
c_{2}(X) \cdot E=c_{2}(E)-K_{E}^{2}=4(g-1) \leq 4(K-1) .
$$

So the first estimate of $(4.2)$ holds if we take $4(K-1)$ as $N$. The second one of (4.2) is already guaranteed by (4.4). Thus, the result follows from (4.2).

Remark. In order to prove finiteness for $\varphi_{i}$ with $E_{i}$ smooth but not necessarily minimal, we see by the same argument that we need to bound $b_{2}\left(E_{i}\right)$.

\section{Proof of Main Theorem 2.}

In this section we prove Main Theorem 2.

We shall make use of the following Theorem proved by Kollár in [4, appendix].

Theorem (5.1). Let $V$ be a Fano manifold with $n=\operatorname{dim} V \geq 4$. Let $X \in\left|-K_{V}\right|$ be a smooth member. Then the inclusion map

$$
i_{*}: \overline{N E}(X) \rightarrow \overline{N E}(V)
$$

is bijective.

Since $\overline{N E}(V)$ is a finite rational polyhedral cone by the cone Theorem, it follows that $\overline{N E}(X)$ is a finite rational polyhedral cone. Dualizing this isomorphism, the nef cones of $V$ and $X$ coincide via restriction and $\overline{A m p}(X)(\simeq \overline{A m p}(V))$ is a finite rational polyhedral cone. Now, in order to prove main Theorem 2 , it is sufficient to show that $c_{2}(X) \cdot D>0$ for all integral divisors $D$ in $\partial \overline{A m p}(X)-\{0\}$, the boundary of the nef cone.

So, assuming that there is an integral divisor $D$ in $\partial \overline{A m p}(X)-\{0\}$ with $D \cdot c_{2}(X)=0$, we shall derive a contradiction.

Denote by $\tilde{D}$ the unique extension of $D$ to $V$. This is nef but not ample on $V$. Replacing $D$ by $m D$, and using the Base Point Free Theorem ([11]), we may assume that $\tilde{D}$ is generated by global sections. Let $\varphi: V \rightarrow W$ be the associated algebraic fiber space. Then $W$ is normal and $\varphi$ has connected fibers. Let $Y=\varphi(X)$ and $\psi=\varphi \mid X$. Note that $\psi$ is not necessarily an 
algebraic fiber space. Since $X$ is an ample divisor in $V$, we have $W=Y$ if $\operatorname{dim} W \leq 3$.

Denoting by $\nu(X, D)$ the numerical Kodaira dimension of $D$, we shall treat the following three cases separately.

Case 1. $\nu(X, D)=1$,

Case 2. $\nu(X, D)=2$,

Case 3. $\nu(X, D)=3$.

Case 1. Here we have $W=Y=\mathbb{P}^{1}$ by $h^{1}\left(\mathcal{O}_{X}\right)=0$. For general $t \in W$ consider $V_{t}=\varphi^{-1}(t)$ and $X_{t}=\psi^{-1}(t)$. Then $V_{t}$ is a smooth Fano threefold with an ample divisor $X_{t} \subset V_{t}$. In particular $X_{t}$ is a smooth connected simply connected surface with trivial canonical class (by the adjunction formula), hence a K3-surface. On the other hand, we have $c_{2}\left(X_{t}\right)=c_{2}(X) \cdot X_{t}=0$, contradiction.

Case 2. Here $Y=W$ is a normal projective surface. Take a general smooth hyperplane section $C \subset W$ such that $C, V_{C}:=\varphi^{-1}(C)$ and $X_{C}:=\psi^{-1}(C)$ are all smooth. Set $f:=\left.\varphi\right|_{V_{C}}: V_{C} \rightarrow C$ and $g:=\left.\psi\right|_{X_{C}}: X_{C} \rightarrow C$. Since $-K_{V_{C}}=-\left.\left(K_{V}+\varphi^{*}(C)\right)\right|_{V_{C}},-K_{V_{C}}$ is $f$-ample. Since $X_{C}=V_{C} \cap X$ and $X_{P}=V_{P} \cap X$ for each $P \in C$, the ampleness of $X$ implies $g: X_{C} \rightarrow C$ is an algebraic fiber space, that is, each fiber of $g$ is connected. Since $c_{2}(X) \cdot D=0$, we know by [17] that $g$ is a (relatively minimal) elliptic fibration with no singular fibers. Thus, by Lemma (2.7), we find an étale covering $\pi: \tilde{C} \rightarrow C$ such that $h^{1}\left(\mathcal{O}_{X_{\tilde{C}}}\right)=h^{1}\left(\mathcal{O}_{\tilde{C}}\right)+1$. Here we set $X_{\tilde{C}}:=X_{C} \times_{C} \tilde{C}$. Let $\pi_{X}: X_{\tilde{C}} \rightarrow X_{C}$ and $\tilde{g}: X_{\tilde{C}} \rightarrow \tilde{C}$ be the natural morphisms. Similarly, set $V_{\tilde{\tilde{C}}}:=V_{C} \times{ }_{C} \tilde{C}$ and denote the natural morphisms by $\pi_{V}: V_{\tilde{C}} \rightarrow V_{C}$ and $\tilde{f}: V_{\tilde{C}} \rightarrow \tilde{C}$. Since $-K_{V_{C}}$ is $f$-ample and since $\pi_{V}$ is étale, $-K_{V_{\tilde{C}}}=$ $\pi_{V}^{*}\left(-K_{V_{C}}\right)$ is also $\tilde{f}$-ample. Thus, the relative version of the KawamataViehweg vanishing theorem ([11]) implies that $R^{j} \tilde{f}_{*} \mathcal{O}_{V_{\tilde{C}}}=0$ if $j>0$ (and $\left.\tilde{f}_{*} \mathcal{O}_{V_{\tilde{C}}}=\mathcal{O}_{\tilde{C}}\right)$. Hence, by the Leray's spectral sequence, we get $h^{1}\left(\mathcal{O}_{V_{\tilde{C}}}\right)=$ $h^{1}\left(\mathcal{O}_{\tilde{C}}\right)$. On the other hand, since $X_{C}$ is ample on $V_{C}$ and $\pi_{V}$ is finite, $X_{\tilde{C}}=\pi^{*} X_{C}$ is an ample divisor on $V_{\tilde{C}}$. Note also that $X_{\tilde{C}}$ and $V_{\tilde{C}}$ are smooth because $\pi_{X}$ and $\pi_{V}$ are étale. Thus, by the Lefschetz theorem, we get $h^{1}\left(\mathcal{O}_{X_{\tilde{C}}}\right)=h^{1}\left(\mathcal{O}_{V_{\tilde{C}}}\right)$ and then $h^{1}\left(\mathcal{O}_{X_{\tilde{C}}}\right)=h^{1}\left(\mathcal{O}_{\tilde{C}}\right)$. But this contradicts the previous equality $h^{1}\left(\mathcal{O}_{X_{\bar{C}}}\right)=h^{1}\left(\mathcal{O}_{\tilde{C}}\right)+1$. 
Case 3. Since $D^{3}>0$ and the linear subsystem $\mid \tilde{D} \|_{X}$ of $|D|$ is free, we have $\operatorname{dim} Y=3$. Let $f: X \rightarrow Z$ be the Stein factorisation of $\psi$. Since $f$ is given by $n D$ for some $n$ and since $D \cdot c_{2}(X)=0$, we obtain $c_{2}(Z)=0$ as a linear form on $\operatorname{Pic}(Z)$ (defined by the projection formula via $f$ ). Hence $Z$ is a quotient of an abelian threefold $A$ by a finite group acting freely in codimension two by [21]. Denote this quotient by $\pi: A \rightarrow Z$. By the complete classification of these quotients given in [18], we have $\rho(Z)=3$ or 9 , in particular $\rho(Z) \geq 2$. Recall that the ample cone $\overline{A m p}(X)$ is a finite rational polyhedral cone ((5.1)). Then the same holds for $\overline{A m p}(Z)$ since this can be regarded as a face of $\overline{A m p}(X)$. Thus we can pick a non-zero rational element $y \in \partial \overline{A m p}(Z)$. Then $\pi^{*}(y) \in \partial \overline{A m p}(A)$. Note that every nef and big divisor on an abelian variety is necessarily ample, because there are no rational curves on an abelian variety. Thus,

$$
0=\pi^{*}(y)^{3}=\operatorname{deg}(\pi) \cdot y^{3} .
$$

This implies $y^{3}=0$. Let $x=f^{*}(y)$. Then $x$ is a non-zero rational point of $\partial \overline{A m p}(X)$ and $x^{3}=x \cdot c_{2}(X)=0$ (for the last equation note that for every divisor $H$ on $Z$ we have $f^{*}(H) \cdot c_{2}(X)=0$ ). In other words, we have a non-zero nef divisor $F$ on $X$ with $F^{3}=0$ and $F \cdot c_{2}(X)=0$. But this is impossible by previous cases 1 and 2 .

This completes the proof of main Theorem 2.

In order to actually get examples of Calabi-Yau threefolds with positive second Chern class via Main Theorem 2, we need to know whether aniticanonical linear system of a smooth Fano fourfold contains smooth members or not. We conclude this section by remarking a few facts concerning this. We specify to the case of Fano fourfolds $V$ of index 2, the case of larger index being obvious. Write $-K_{V}=2 H$. If $b_{2}(V)=1$, Wilson proved the existence of smooth members in $|H|$, hence by Mukai's classification [15], $-K_{V}$ is even generated by global sections. The same holds for $b_{2}(V) \geq 2$ due to the classification given by Theorem (5.2) below (without the assumption on smooth members in $H$ ). Hence Main Theorem 2 applies to the general member of the anti-canonical system on every Fano fourfold of index 2. At present no direct proof on the existence of smooth members of the anticanonical system of a Fano fourfold of index 2 and $b_{2} \geq 2$ is known. In case of index 1 , nothing is known about the existence of smooth members in the anti-canonical system.

Theorem (5.2). The following is a complete list of all Fano fourfolds $V$ with index 2 and $b_{2}(V) \geq 2$. 
(1) $\mathbb{P}^{1} \times W$ with $W$ a Fano threefold of index 2 or 4 ,

(2) a 2:1 - covering over $\mathbb{P}^{2} \times \mathbb{P}^{2}$ ramified over a divisor of degree (2,2),

(3) a divisor of type $(1,2)$ in $\mathbb{P}^{2} \times \mathbb{P}^{3}$,

(4) a linear section of $\mathbb{P}^{2} \times Q_{3}$,

(5) the projectivisation of the nullcorrelation bundle over $\mathbb{P}^{3}$,

(6) a linear section in $\mathbb{P}^{3} \times \mathbb{P}^{3}$,

(7) a linear section in the Fano fivefold obtained by blowing up $\mathbb{P}^{5}$ along a line,

(8) the blow up of $Q_{4}$ along a conic with the additional requirement that the plane spanned by the conic in $\mathbb{P}^{5}$ is not in $Q_{4}$,

(9) $\mathbb{P}\left(\mathcal{O}_{\mathbb{P}^{3}}(2) \oplus \mathcal{O}_{\mathbb{P}^{3}}\right)$, and

(10) $\mathbb{P}\left(\mathcal{O}_{Q_{3}}(1) \oplus \mathcal{O}_{Q_{3}}\right)$.

Proof. This follows from several papers, but is never stated in the literature explicitly. A complete argument here has kindly been given to us by J. Wisniewski [32]. For more details, see [29], the references given there and [2]. Q.E.D.

Remark. (1) Assuming the existence of smooth members, this classifcation was already done in [15] and [29].

(2) In all the cases (2) - (10) we have $b_{2}(V)=2$, so there are exactly two Mori contractions on $V$. In the cases (2) - (6) both of these are of fiber type but not birational. This is obvious except in (5). In this case the second contraction is given by the projection $V=\mathbb{P}(F)$, where $F$ is a so-called spinor bundle over the quadric $Q_{3}$. We refer to [19]. In cases (7) - (10) one contraction is birational and the other is of fiber type.

(3) Of course, it is just a matter of (tedious) calculations to verify main Theorem 2 in the case of Fano fourfolds of index 2 using Theorem (5.2).

\section{References.}


[1] V. A. Alexeev, Boundedness and $K^{2}$ for log surfaces, Intern. J. Math. 5 (1995), 779-810.

[2] M. Andreatta and J. Wisniewski, A note on non-vanishing and applications, Duke Math. J. 72 (1993), 739-755.

[3] W. Barth, C. Peters, and A. Ven de Van, Compact complex surfaces, Springer 1984.

[4] C. Borcea, Homogeneous vector bundles and families of Calabi-Yau threefolds II, Proc. Symp. Pure Math. 52 (1991), (Part II) 83-91.

[5] L. Brenton, On singular surfaces with negative canonical bundle, Math. Ann. 248 (1981), 117-124.

[6] M. Gross, A finiteness theorem for elliptic Calabi-Yau threefolds, Duke Math. J. 74 (1994), 271-299.

[7] A. Grassi and D. R. Morrison, Automorphisms and the Kahler cone of certain Calabi-Yau manifolds, Duke Math. J. 71 (1993), 831-838.

[8] F. Hidaka and K, Watanabe, Normal Gorenstein surfaces with ample anticanonical divisor, Tokyo J. Math. 4 (1981), 319-330.

[9] Y. Kawamata, Kodaira dimensions of certain algebraic fiber spaces, J. Fac. Sci. Univ. Tokyo Sect IA Math. 30 (1983), 1-24.

[10] Y. Kawamata, Minimal models and the Kodaira dimension of algebraic fiber spaces, Crelles J. 363 (1985), 1-46.

[11] Y. Kawamata, K. Matsuda and K. Matsuki, Introduction to the minimal model problem, Adv. St. Pure Math. 10 (1987), 283-360.

[12] J. Kollár, Flops, Nagoya Math. J. 113 (1989), 15-36.

[13] Y. Miyaoka, The Chern classes and Kodaira dimension of a minimal variety, Adv. Stud. Pure Math. 10 (1987), 449-476.

[14] D. Morrison, Compactifications of moduli spaces inspired by mirror symmetry, Asterisque, 218 (1993), 243-271.

[15] S. Mukai, Fano manifolds of coindex 3, Proc. Natl. Acad. Sci. USA, 86 (1990), 3000-3002.

[16] N. Nakayama, On Weierstrass models, in Algebraic Geometry and Commutative Algebra: in Honor of Masayoshi Nagata, Kinokuniya, Tokyo, 1988, 405-431.

[17] K. Oguiso, On algebraic fiber space structures on a Calabi-Yau 3-fold, Intern. J. Math. 4 (1993), 439-465. 
[18] K. Oguiso, On the complete classification of Calabi-Yau threefolds of Type $I I I_{0}$, Higher Dimensional Complex Varieties, Proc. Trento 1994, 328-340. Ed. M. Andretta, T. Peternell. de Gruyter 1996.

[19] T. Peternell, M. Szurek and J. Wisniewski, Numerically effective vector bundles with small Chern classes, Proceedings Algebraic Geometry Bayreuth 1990, Lecture Note in Math. 1507 (1991), 145-156.

[20] M. Reid, Minimal models of canonical 3-folds, Adv. Stud. Pure Math. 1 (1983), 395-418.

[21] N.I. Shepherd-Barron and P.M.H. Wilson, Singular threefolds with numerically trivial first and second Chern class, J. Alg. Geom. 3 (1994), 265-281.

[22] T. Shioda and H. Inose, On singular K3 surfaces, in Complex analysis and algebraic geometry, Iwanami shoten 1977, 119-136.

[23] Utah, Utah seminar on July 1994.

[24] P.M.H. Wilson, Calabi-Yau manifolds with large Picard number, Invent. Math. 98 (1989), 139-155.

[25] P.M.H. Wilson, The Kähler cone on Calabi-Yau threefolds, Invent. Math. 107, 114, 231-233 (1993), 139-155.

[26] P.M.H. Wilson, Minimal models of Calabi-Yau threefolds, in Classification of Algebraic Varieties, L'Aquila 1992, Contemporary mathematics 162 (1994), 403-410.

[27] P.M.H. Wilson, The role of $c_{2}$ in Calabi-Yau classification- a preliminary survey, preprint 1994.

[28] P.M.H. Wilson, Symplectic deformations of Calabi-Yau threefolds, preprint 1995.

[29] J. Wisniewski, Fano 4-folds of index 2 with $b_{2} \geq 2$. A contribution to Mukai Classification, Bull. Polish Acad. Sci. Math. 38 (1990), 173-183.

[30] J. Wisniewski, On contractions of extremal rays of Fano manifolds, Crelles J. 417 (1991), 141-157.

[31] J. Wisniewski, A report on Fano manifolds of middle index and $b_{2} \geq 2$, preprint 1993.

[32] J. Wisniewski, A letter to the authors, 1994. 
RECEIVED JUly 2, 1996.

UNIVERSITY OF TOKYO

KOMABA, TOKYO, JAPAN

AND

UNIVERSITÄT BAYREUTH

D-95440 Bayreuth, Germany 